\title{
The origin of retrograde hot Jupiters
}

\author{
Smadar Naoz ${ }^{1}$, Will M. Farr ${ }^{1}$, Yoram Lithwick ${ }^{1}$, Frederic A. Rasio ${ }^{1}$ \\ and Jean Teyssandier ${ }^{1}$ \\ ${ }^{1}$ Center for Interdisciplinary Exploration and Research in Astrophysics (CIERA), \\ Northwestern University, Evanston, IL 60208, USA \\ email: snaoz@northwestern.edu
}

\begin{abstract}
Many hot Jupiters are observed to be misaligned with respect to the rotation axis of the star (as measured through the Rossiter-McLaughlin effect) and some (about $\sim 25 \%$ ) even appear to be in retrograde orbits. We show that the presence of an additional, moderately inclined and eccentric massive planet in the system can naturally explain close, inclined, eccentric, and even retrograde orbits. We have derived a complete and accurate treatment of the secular dynamics including both the key octupole-order effects and tidal friction. The flow of angular momentum from the inner orbit to the orbit of the perturber can lead to both high eccentricities and inclinations, and even flip the inner orbit. In our treatment the component of the inner orbit's angular momentum perpendicular to the stellar equatorial plane can change sign; a brief excursion to very high eccentricity during the chaotic evolution of the inner orbit can then lead to rapid "tidal capture," forming a retrograde hot Jupiter. Previous treatments of the secular dynamics focusing on stellar-mass perturbers would not allow for such an outcome, since in that limit the component of the inner orbit's angular momentum perpendicular to the stellar equatorial plane is strictly conserved. Thus, the inclination of the planet's orbit could not change from prograde to retrograde.
\end{abstract}

Keywords. planets and satellites: formation, planetary systems: formation

\section{Introduction}

The search for extrasolar planets has led to the surprising discovery of many Jupiterlike planets in very close proximity to their host star (e.g., Nelson 2001), the so-called "hot Jupiters" (hereafter HJ). Even more surprisingly, many of these hot Jupiters have orbits that are eccentric or highly inclined with respect to the rotation axis of the star (as measured through the Rossiter-McLaughlin effect (Gaudi \& Winn 2007)) and some (8 out of 32) appear to be in retrograde orbits (Triaud et al. 2010). How they get so close to the star in such orbits remains an open question. Slow migration though a protoplanetary disk (e.g., Lin \& Papaloizou 1986 and Masset \& Papaloizou 2003) would produce orbits with low eccentricities and inclinations. However, this mechanism cannot explain the recent observations. Some models, such as those of Fabrycky \& Tremaine (2007) and Wu et al. (2007) invoke a companion star in the system, which perturbs the inner orbit and can produce increases in eccentricity and inclination but not retrograde orbits, i.e., the inclination of the inner orbit with respect to the total angular momentum cannot change sign.

\section{Secular interaction between two giant planets}

We define the orientation of the inner orbit so that a prograde (retrograde) orbit has $i_{1}<90^{\circ}\left(i_{1}>90^{\circ}\right)$, where $i_{1}$ is the inclination of the inner orbit with respect to the total angular momentum, assumed parallel to the stellar rotation axis. We note that 
the directly observed parameter is actually the projected angle between the spin axis of the star and the planet's angular momentum. This angle can be above $90^{\circ}$ even if $i_{1}$ is prograde (i.e., below, but close to $90^{\circ}$; see Fabrycky \& Tremaine 2007, and also Fig. 1(c)). However, for simplicity we focus here on the physical angle $i_{1}$ (see section 4 for discussion and comparison with observations).

We assume a hierarchical configuration, where the star and Jupiter-like planet form a relative tight binary, and with the outer planet in a much wider orbit than the inner one. In the secular approximation the orbits may change shape and orientation but the semi-major axes (SMA) are strictly conserved in the absence of tidal dissipation (e.g., Ford et al. 2000; Eggleton et al. 1998). In particular, the Kozai-Lidov mechanism (Kozai 1962; Lidov 1962) produces large-amplitude oscillations of the eccentricity and inclination when the initial relative inclination between the inner and outer orbits is sufficiently large $\left(40^{\circ} \lesssim i \lesssim 140^{\circ}\right.$, for initial eccentricity of the inner orbit close to zero). We have derived the secular evolution equations to octupole order using Hamiltonian perturbation theory (e.g., Kozai 1962; Harrington 1969; Krymolowski \& Mazeh 1999; Ford et al. 2000). The octupole-level terms can give rise to fluctuations in the eccentricity maxima to arbitrarily high values (see Krymolowski \& Mazeh 1999; Ford et al. 2000; Blaes et al. 2002 ), in contrast to the regular evolution in the quadrupole potential, where the amplitude of eccentricity oscillations is constant (e.g., Fabrycky \& Tremaine 2007; Wu et al. 2007; Mazeh \& Shaham 1979).

Unlike previous derivations of "Kozai-type" evolution, our treatment allows for changes in the $z$-components of the orbital angular momenta (i.e., the components along the total angular momentum) $L_{\mathrm{z}, 1}$ and $L_{\mathrm{z}, 2}$ (see Naoz et al. 2010, 2011 for more details). Many previous studies of secular perturbations in hierarchical triples considered a stellar-mass perturber, for which $L_{z, 1}$ is very nearly constant (e.g., Mazeh \& Shaham 1979; Fabrycky \& Tremaine 2007; Wu et al. 2007; Perets \& Naoz 2009). Moreover, the assumption that $L_{\mathrm{z}, 1}$ is constant has been built into previous derivations. However, this assumption is only valid as long as $L_{2} \gg L_{1}$, which is not the case in comparable-mass systems (with two planets). Unfortunately, an immediate consequence of this assumption is that a prograde orbit can never be turned into a retrograde orbit.

\section{Tidal friction}

We adopt the tidal evolution equations of Eggleton, Kiseleva \& Hut (1998), which are based on the equilibrium tide model of Hut (1981). The complete equations can be found in Fabrycky \& Tremaine (2007, eqs A1-A5). Following their approach (see their eq. A10) we set the tidal quality factors $Q_{1,2} \propto P_{\text {in }}$ [see also Hansen 2010, eq. (11)]. This means that the viscous times of the star and planet remain constant; the representative values we adopt here are $5 \mathrm{yr}$ for the star and $1.5 \mathrm{yr}$ for the planet, which correspond to $Q_{\star}=5.5 \times 10^{6}$ and $Q_{J}=5.8 \times 10^{6}$, respectively, for a 1-day period.

In figure 1 we show the secular dynamical evolution of a two-planet system with tidal dissipation. The inner planet becomes retrograde at $82 \mathrm{Myr}$, and remains retrograde after circularizing into a hot Jupiter. During each excursion to very high eccentricity for the inner orbit [marked with vertical lines in panels (b) and (d)], tidal dissipation becomes significant. Eventually the inner planet is tidally captured by the star and its orbit becomes decoupled from the outer body. After this point the orbital angular momenta remain nearly constant. The final SMA for the inner planet is at $0.024 \mathrm{AU}$, typical of a hot Jupiter. We monitor the pericenter distance of the inner planet to ensure that it always remains outside the Roche limit (Matsumura et al. 2010). 

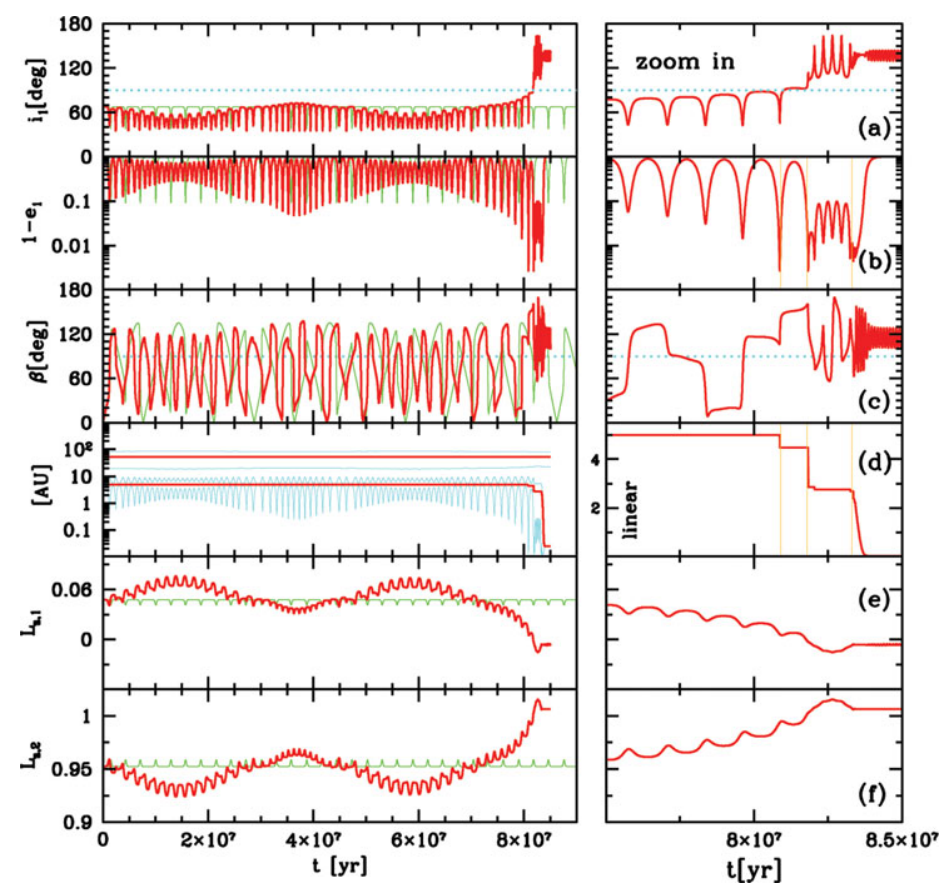

Figure 1. Dynamical evolution of a representative two-planet system with tidal dissipation included. Here the star has mass $1 M_{\odot}$, the inner planet $1 M_{\mathrm{J}}$ and the outer planet $3 M_{\mathrm{J}}$. The inner orbit has SMA $a_{1}=5 \mathrm{AU}$ and the outer orbit has SMA $a_{2}=51 \mathrm{AU}$. The initial eccentricities are $e_{1}=0.001$ and $e_{2}=0.6$ and the initial relative inclination $i=74.5^{\circ}$. We show: (a) the inner orbit's inclination $\left(i_{1}\right)$; (b) the eccentricity of the inner orbit (as $\left.1-e_{1}\right)$; (c) the spin orbit angle $\beta$ (d) the SMA, peri-, and apo-center distances for the inner orbit and the peri- and apo-center distance for the outer orbit; and, in (e) and (f) the $z$-components of the inner and outer orbit's angular momentum, normalized to the total angular momentum. The initial mutual inclination of $74.5^{\circ}$ corresponds to inner- and outer-orbit inclinations of $67.6^{\circ}$ and $6.9^{\circ}$, respectively. The thin curves shows the evolution in the quadrupole approximation (but including tidal friction), demonstrating that the octupole-order effects lead to a qualitatively different behavior.

\section{Discussion - Comparison to observations}

We showed that retrograde HJ orbits can be the result of a dynamical evolution in the presence of an additional moderately inclined and eccentric planet in the system. As mention above the observable parameter from the Rossiter-McLaughlin effect is the projected angle between the star's spin and the orbital angular momentum (the projected obliquity, Gaudi \& Winn 2007). In Fig. 1 we show both the inclination $i_{1}$ [panel (a)] and also the angle between the star's spin axis and the planet's orbit $\beta$ [panel (c)]. We note that, in contrast to the quadrupole order result, the spin orbit angle continues to oscillate at the same rate as the inclination, after the HJ is formed. However, the planet still appears to be in retrograde motion (i.e., $\beta>90^{\circ}$ ). Here instead defined retrograde orbits with respect to the total angular momentum, and we focused on the true angle between the orbital angular momentum of the inner planet and the invariable plane.

Projection effects can cause the true inclination and the spin-orbit angle to differ in magnitude, or even sign. Moreover, several mechanisms have been proposed in the literature that could, under certain assumptions, directly affect the spin axis of the star. These mechanisms can re-align the stellar spin axis through tidal interactions with either a slowly spinning star (Matsumura et al. 2010) or with the outer convective layer of a 
sufficiently cold star (Winn et al. 2010b). Additionally, a magnetic interaction between the star and a significantly charged protoplanetary disk with negligible accretion could also lead to misalignment between the stellar spin and the disk (Lai et al. 2011).

These effects can potentially complicate the interpretation of any specific observation. Nevertheless, if hot Jupiters are produced by the simple mechanism described here, many of their orbits should indeed be observed with large projected obliquities.

\section{References}

Blaes, O., Lee, M. H., \& Socrates, A. 2002, ApJ, 578, 775

Chatterjee, S., Ford, E. B., Matsumura, S., \& Rasio, F. A. 2008, ApJ, 686, 580

Eggleton, P. P., Kiseleva, L. G., \& Hut, P. 1998, ApJ, 499, 853

Fabrycky, D. \& Tremaine, S. 2007, ApJ, 669, 1298

Ford, E. B., Kozinsky, B., \& Rasio, F. A. 2000, ApJ, 535, 385

Gaudi, B. S. \& Winn, J. N. 2007, ApJ, 655, 550

Hansen, B. 2010, ApJ, 723, 285

Harrington, R. S. 1969, Celestial Mechanics, 1, 200

Holman, M., Touma, J., \& Tremaine, S. 1997, Nature, 386, 254

Hut, P. 1998, A\&A, 99, 134

Jefferys, W. H. \& Moser, J. 1966, AJ, 71, 568

Kalas, P., et al. 2008, Science, 322, 1345

Kozai, Y. 1962, AJ, 67, 591

Krymolowski, Y. \& Mazeh, T. 1999, MNRAS, 304, 720

Lai, D., Foucart, F., \& Lin, D. N. C. 2011, MNRAS, 412, 2790

Lidov, M. L. 1962, Planetary and Space Science, 9, 719

Lin, D. N. C. \& Papaloizou, J. 1986, ApJ, 309, 846

Marois, C., et al. 2008, Science, 322, 1348

Masset, F. S. \& Papaloizou, J. C. B. 2003, ApJ, 588, 494

Matsumura, S., Peale, S. J., \& Rasio, F. A. 2010, ApJ, 725, 1995

Mazeh, T. \& Shaham, J. 1979, A\&A, 77, 145

Nagasawa, M., Ida, S., \& Bessho, T. 2008, ApJ, 678, 498

Naoz S., Farr W. M., Lithwick Y., Rasio F. A., \& Teyssandier J. 2010, Nature, 473, 187

Naoz S., Farr W. M., Lithwick Y., \& Rasio, F. A. 2011, in preparation

Nelson, R. P. 2001, Solar and extra-solar planetary systems, 577, 35

Perets, H. B. \& Naoz, S. 2009, ApJ, 699, L17

Pollack, J. B., Hubickyj, O., Bodenheimer, P., Lissauer, J. J., Podolak, M., \& Greenzweig, Y. 1996, Icarus, 124,62

Schlaufman, K. C. 2010, ApJ, 712602

Triaud, A. H. M. J., et al. 2010, A\&BA, 524, A25

Winn, J. N., Fabrycky, D., Albrecht, S., \& Johnson, J. A. 2010, ApJ, 718145

Wu, Y., Murray, N. W., \& Ramsahai, J. M. 2007, ApJ, 670, 820 